\title{
Multiple Myopericytoma of the Face and Parotid Gland
}

\author{
Yun-Ik Jung ${ }^{1}$, Yoon-Kyu Chung ${ }^{2}$, Seum Chung ${ }^{3}$ \\ ${ }^{1}$ Department of Plastic and Reconstructive Surgery, Yonsei University College of Medicine, Seoul; ${ }^{2}$ Department of Plastic and Reconstructive \\ Surgery, Yonsei University Wonju College of Medicine, Wonju; ${ }^{3}$ Department of Plastic and Reconstructive Surgery, National Health Insurance \\ Corporation Ilsan Hospital, Goyang, Korea
}

Myopericytoma is a benign tumor that is composed of myoid-appearing oval to spindleshaped cells with a concentric perivascular pattern of growth. The tumor is morphologically heterogeneous and can exhibit a broad histologic spectrum. We describe a case of multiple myopericytoma occurring in the head and neck skin region with involvement of the parotid gland where it is known to occur very rarely. A 40-year-old woman noticed multiple enlarging, painless, round-shaped masses on her left cheek. The patient had experienced a similar lesion of the same area 8 years earlier which was completely excised and the pathological diagnosis was spindle cell type myoepithelioma. On a computed tomographic image, one mass involved the superficial parotid gland and was well encapsulated. Excision of the facial masses and superficial parotidectomy with facial nerve preservation were performed. A diagnosis of myopericytoma was established in light of the immunohistochemical pattern with the histopathological findings. Over the 4-year follow-up period, there was no evidence of recurrence. As many perivascular myoid neoplasms share common morphologic features with myopericytoma, we should consider the differential diagnosis, and confirm the histological findings with appropriate immunohistochemical staining. After identifying myopericytoma, it should be treated with wide surgical excision to prevent local recurrence.

Keywords Head and neck neoplasms / Parotid neoplasms / Parotid gland

\author{
Correspondence: Seum Chung \\ Department of Plastic and \\ Reconstructive Surgery, National \\ Health Insurance Corporation Ilsan \\ Hospital, 100 Ilsan-ro, llsandong-gu, \\ Goyang 410-719, Korea \\ Tel: +82-31-900-0560 \\ Fax: +82-31-900-0343 \\ E-mail: schung@nhimc.or.kr
}

This article was presented as a poster at the 69th Congressof the Korean Society of Plastic and Reconstructive Surgeons on November 11-13, 2011

No potential conflict of interest relevant to this article was reported.

Received: 1 Oct $2011 \bullet$ Revised: 1 Nov 2011 • Accepted: 2 Nov 2011

pISSN: 2234-6163 • elSSN: 2234-6171 • http://dx.doi.org/10.5999/aps.2012.39.2.158• Arch Plast Surg 2012;39:158-161

\section{INTRODUCTION}

Myopericytoma is described as a benign tumor that is composed of myoid-appearing oval to spindle-shaped cells with a concentric perivascular pattern of growth. The term myopericytoma was first proposed by Requena et al. [1] as an alternative designation for solitary myofibroma derived from myopericytes. The concept of perivascular myoid differentiation was established by Granter et al. [2]. They proposed the designation perivascular myoma to describe a spectrum of neoplasms exhibiting mor- phologic and immunohistochemical evidence of perivascular myoid differentiation [2]. In 2002, the World Health Organization suggested the use the term of myopericytoma.

The tumor is morphologically heterogeneous and can exhibit a broad histologic spectrum, so that myopericytoma is clinically and morphologically distinct from other entities such as hemangiopericytoma, myofibroma, glomangiopericytoma, and angioleiomyoma $[3,4]$. In this report, we describe a case of multiple myopericytoma occurring in the head and neck skin region with involvement of the parotid gland, where it is known to occur 
very rarely.

\section{CASE}

A 32-year-old woman noticed a slowly enlarging, painless, isolated, round mass on her left cheek. Excision of the mass was performed. Grossly, the mass was localized within the subcutaneous tissue and measured $20 \times 17 \mathrm{~mm}$. It was completely excised, and the pathological diagnosis was spindle cell type myoepithelioma.

Eight years later, she again noticed multiple masses in the left facial area. Physical examination revealed freely movable solid masses without tenderness or ulceration. The regional lymph nodes were not palpable (Fig. 1A). A computerized tomography scan disclosed a mass in the superficial parotid gland tissue and

\section{Fig. 1. Case}

(A) A 32-year-old woman noticed multiple masses on her left facial area. (B) After superficial parotidectomy, the tumor was completely excised and the facial nerve was well preserved.
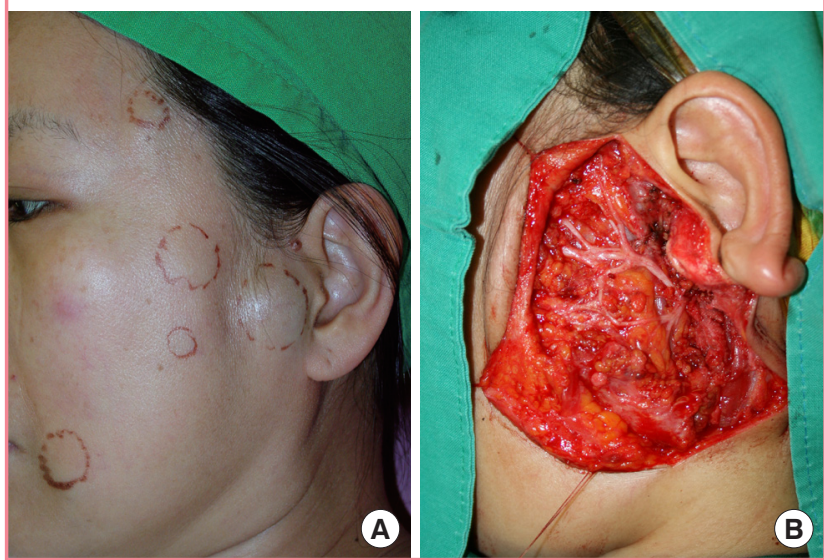

Fig. 2. Case

A computerized tomography scan disclosed the mass measuring $22 \mathrm{~mm}$ diameter in the left parotid gland. Small multiple masses were also found in the subcutaneous layer of the left facial area.

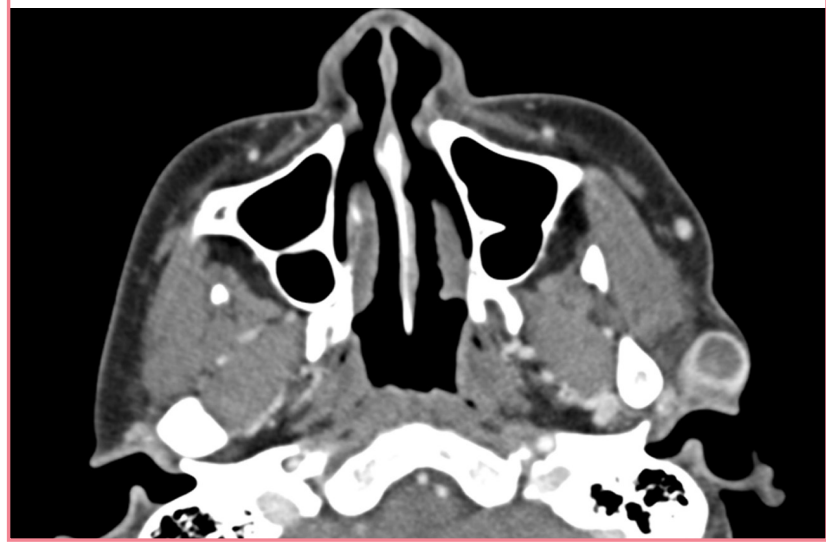

eight well capsulated masses in the left facial area. They measured from 8 to $22 \mathrm{~mm}$ in diameter (Fig. 2). Excision of the facial masses and superficial parotidectomy with facial nerve preservation were performed (Fig. 1B). The specimens were well circumscribed, nodular, and rubbery in consistency. A cut section of the parotid gland mass revealed a subcapsular, round, reddish-tan, solid, firm mass measuring $25 \times 20 \times 20 \mathrm{~mm}$ (Fig. 3).

Histological findings of the tumors were characterized as capsulation by collagenous fibers and the presence of oval-shaped cells with eosinophilic cytoplasm arranged around the numerous vessels (Fig. 4). The perivascular myoepithelial cells expressed positive reactivity for smooth muscle actin staining. CD31 staining had shown expression only of endothelial cells of the surrounding vessels without the presence of neoplastic cells (Fig. 5). We concluded that the pathological diagnosis of all the masses on the face and parotid gland was myopericytoma.

\section{DISCUSSION}

Myopericytoma is an uncommon tumor, so reports of myopericytoma are very rare and therefore surgeons and pathologists have difficulty readily making a diagnosis. Myopericytoma is generally considered a slow-growing benign tumor and the diameter is usually less than $2 \mathrm{~cm}$. It occurs predominantly in the skin and superficial soft tissue of the distal extremities, followed by the head and neck region, and the trunk. It could be located multifocally in an anatomic area. It occurs very rarely in the different organs of the head and neck region with multiple large features. The tumor may recur locally and its recurrence is noted in rare cases $[4,5]$.

Myopericytoma was recently characterized as a soft-tissue neoplasm with perivascular differentiation of myoid cells. Dictor

\section{Fig. 3. Case}

On cut section of the parotid gland mass, it revealed a subcapsular round shaped reddish tan colored solid firm mass measuring $25 \times 20$ $\times 20 \mathrm{~mm}$. 
Fig. 4. Microscopic findings with H\&E

(A) The tumor was located in the superficial parotid gland and was well encapsulated $(\times 4)$. (B) The presence of oval-shaped cells with eosinophilic cytoplasm arranged around the numerous vessels $(x 100)$.
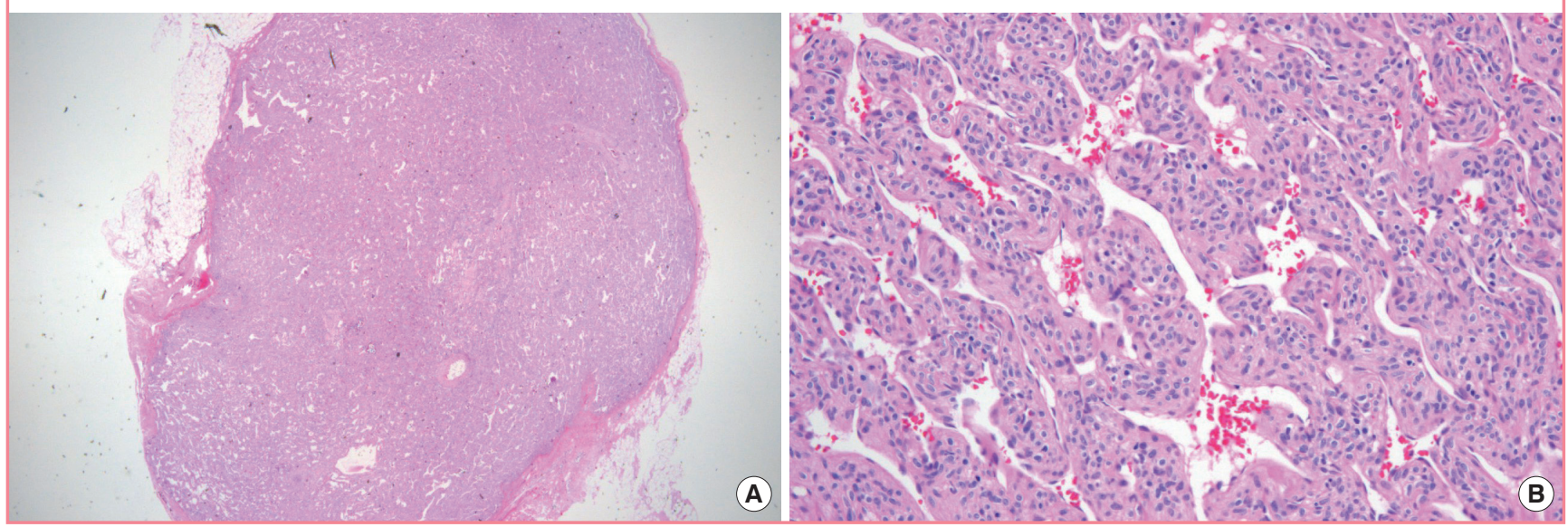

Fig. 5. Immunohistochemical staining examinations

(A) The perivascular myoepithelial cells expressed positive reactivity for smooth muscle actin staining $(\times 200)$. (B) CD31 staining had expressed only for endothelial cells of the surrounding vessels without the presence of neoplastic cells $(\times 200)$.

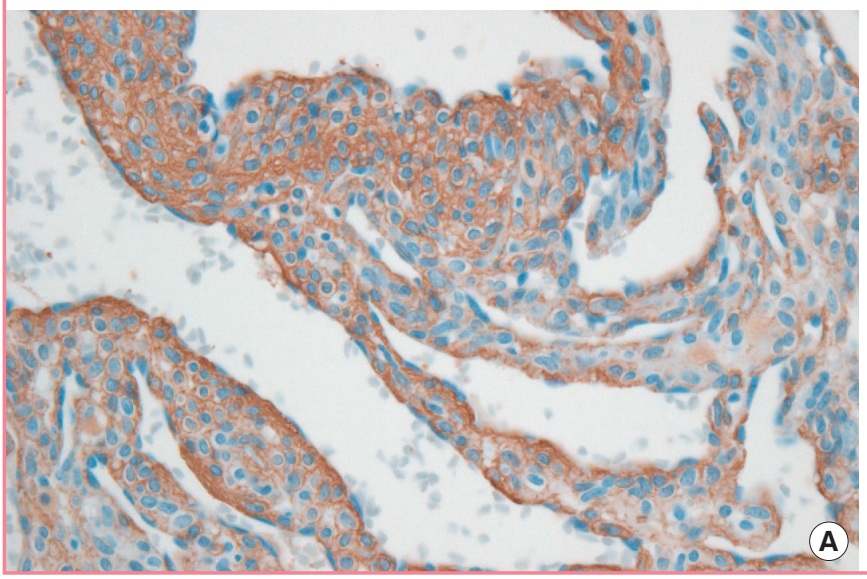

et al. [6] described the term myopericyte by a case of hemangiopericytoma with a myofibromatous pattern. It represents a transitional form between pericytes and the smooth muscle cells of vessels. Microscopic examination of a benign myopericytoma with hematoxylin-eosin-staining is characterized by collagenous fibrous capsulation and the presence of cells arranged inside concentric islands. The tumor is also characterized by the presence of surrounding numerous small to medium-sized vessels with a concentric perivascular arrangement of neoplastic cells, presenting even brand of cell and exhibited ovoid nuclei, even distributed chromatin, and distinct nucleoli with abundant eosinophilic cytoplasm $[3,5]$.

The neoplastic cells exhibited strong and diffuse immunoreactivity for muscle specific actin, smooth muscle actin (SMA), CD34, and bcl-2, whereas the tumor was negative for keratin, epithelial membrane antigen, desmin, S-100 protein, human

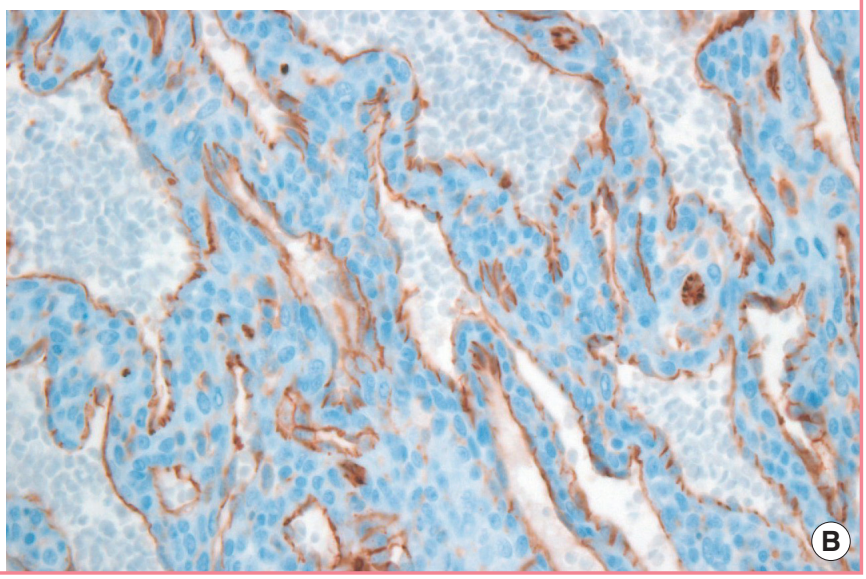

melanoma black-45, Melan-A, CD31, chromogranin, and synaptophysin $[1,3]$.

A diagnosis of myopericytoma was established along the immunohistochemical pattern with the histopathological findings. A contrast-enhanced computed tomography (CT) examination is thought to be helpful for evaluation and in the differential diagnosis for myopericytoma. Peripheral enhancement, irregular central non-enhanced attenuation masses with unsmoothed margins and single or multiple slow-growing reactive lymph nodes are the CT features of myopericytoma, but they are nonspecific [7].

Myopericytoma of the parotid gland should be distinguished from hemangiopericytoma and myoepithelioma because these benign tumors are similar in histological features and occur rarely in the parotid gland.

Hemangiopericytoma has a perivascular arrangement of ovoid cells with expression of myogenic markers, but it is characterized 
by staghorn-shaped spaces with an intervening proliferation of cells and rarely, it may show immunoreactivity to SMA and consistent positivity for $\mathrm{CD} 34$ staining $[5,8]$.

Myoepithelioma shows nonspecific histopathologic features but its most common variant is the spindle cell type. It is characterized by myoepithelial cells, contains few ducts, and may show strongly positive for calponin and S-100 protein but not for vimentin, cytokeratins, or SMA [8].

Patients with benign myopericytomas are usually treated with complete surgical excision. In the parotid gland myopericytoma specifically, the superficial parotidectomy with facial nerve preservation is known as a treatment of choice [9]. The prognosis of this entity is good. In benign cases, local recurrence occurs rarely. A malignant myopericytoma is extremely rare, often with local recurrence and distant metastases in other organs or extensive metastases, and the metastasizing neoplasm is associated with the depth of the neoplasm $[3,4]$. In this case, the lesion occurred locally in the multifocal region, but it is not clear whether the tumor that occurred in the parotid gland is a recurrent one or not. It was probably due to an incomplete excision of the tumor or the characteristic multifocal feature. Over the 4 year follow-up period, there was no evidence of recurrence.

As many perivascular myoid neoplasms share common morphologic features with myopericytoma, we should consider the differential diagnosis, and confirm the histological findings with appropriate immunohistochemical staining. After identifying myopericytoma, it should be treated with wide surgical excision to prevent local recurrence.

\section{REFERENCES}

1. Requena L, Kutzner H, Hugel H, et al. Cutaneous adult myofibroma: a vascular neoplasm. J Cutan Pathol 1996;23: 445-57.

2. Granter SR, Badizadegan K, Fletcher CD. Myofibromatosis in adults, glomangiopericytoma, and myopericytoma: a spectrum of tumors showing perivascular myoid differentiation. Am J Surg Pathol 1998;22:513-25.

3. Lau SK, Klein R, Jiang Z, et al. Myopericytoma of the kidney. Hum Pathol 2010;41:1500-4.

4. Mentzel T, Dei Tos AP, Sapi Z, et al. Myopericytoma of skin and soft tissues: clinicopathologic and immunohistochemical study of 54 cases. Am J Surg Pathol 2006;30:104-13.

5. Xia L, Chen Y, Geng N, et al. Multifocal myopericytoma in the maxillofacial region: a case report. Oral Surg Oral Med Oral Pathol Oral Radiol Endod 2010;109:e59-62.

6. Dictor M, Elner A, Andersson T, et al. Myofibromatosis-like hemangiopericytoma metastasizing as differentiated vascular smooth-muscle and myosarcoma. Myopericytes as a subset of "myofibroblasts". Am J Surg Pathol 1992;16:1239-47.

7. Chu ZG, Yu JQ, Yang ZG, et al. Myopericytoma Involving the parotid gland as depicted on multidetector CT. Korean J Radiol 2009;10:398-401.

8. Yaman H, Gerek M, Tosun F, et al. Myoepithelioma of the parotid gland in a child: a case report. J Pediatr Surg 2010; 45:E5-7.

9. Song JW, Choi HJ, Kim MS, et al. Tumors of the parotidomasseteric area associated with inadequate primary treatment: report of 2 cases. J Korean Soc Plast Reconstr Surg 2006;33:764-8. 